\section{Audit of small-incision cataract surgery using an anterior chamber maintainer}

T. SHARMA, N. DHINGRA, T. WORSTMANN

\begin{abstract}
Purpose To assess the results of small-incision cataract surgery with intraocular lens implantation using an anterior chamber maintainer (ACM) performed between March 1997 and December 1998.

Method A retrospective audit was performed of all 300 consecutive patients who underwent extracapsular cataract extraction using a $6 \mathrm{~mm}$ scleral tunnel incision, anterior chamber maintainer and manual fragmentation of the nucleus.

Results Ninety per cent of patients had a gain in visual acuity at the end of 3 months. The rate of posterior capsule opacification was comparable to the results of the National Cataract Surgery Survey (RCO 1993), i.e. 13\%, but the rate of corneal endothelial decompensation and endophthalmitis was marginally higher.

Conclusion Appropriate selection of cases, meticulous wound closure and subconjunctival antibiotics at the end of surgery make this an acceptable alternative small-incision closed-system low-cost procedure where phacoemulsification is not available.
\end{abstract}

Key words Anterior chamber maintainer, Cataract, Complications, Extracapsular

An anterior chamber maintainer was recommended by Blumenthal and Moisseiev ${ }^{1}$ to

T. Sharma

N. Dhingra

T. Worstmann

Queen's Hospital

Burton-on-Trent, UK

Miss T. Worstmann

Queen's Hospital

Burton-on-Trent

Staffordshire DE13 ORB, UK

Fax: +44 (0)1283-593014

e-mail:

ndhingra99@yahoo.com

Received: 17 May 1999 Accepted in revised form: 9 December 1999 facilitate manipulation in the anterior chamber during cataract surgery. Subsequently the combination of a three-step scleral tunnel incision, anterior chamber maintainer and manual fragmentation of the nucleus allowed the development of a low-tech, low-cost cataract extraction technique with intraocular lens (IOL) implantation which had all the advantages of a small-incision closed-system procedure. We present an audit of 300 consecutive cases of extracapsular cataract extraction with IOL implantation using a modified 'MiniNuc' technique, as described by Blumenthal and Moisseiev, ${ }^{1}$ performed between March 1997 and
December 1998. The results were compared with those of the National Cataract Surgery Survey (NCSS) performed by the Royal College of Ophthalmologists in 1993.

\section{Materials and methods \\ Surgical technique}

After placing a superior rectus traction suturea self-retaining anterior chamber maintainer (ACM) was placed through a limbal paracentesis in the inferior temporal quadrant. The ACM was connected to a $500 \mathrm{ml}$ bag of Hartman's solution containing adrenaline 1:1000 (0.5 ml). A three-step scleral tunnel ${ }^{2}$ incision was made at 12 o'clock using a diamond knife, crescent blade and phacoknife.

The external incision of the tunnel was approximately $6 \mathrm{~mm}$ long and the tunnel continued well into the clear cornea, to create an internal stromal lip. The internal opening was extended to around $6.5 \mathrm{~mm}$ to allow the nucleus to be engaged. A single side port using an MVR knife was made at 10 o'clock to allow access to the anterior chamber with the self-sealing section closed.

A large capsulorrhexis $(5-6 \mathrm{~mm})$ was performed using a cystitome through the side port. Relieving incisions were given into the capsulorrhexis, where necessary, if it turned out to be smaller than intended. With the ACM closed hydrodissection of the nucleus was performed through the section to dislocate the nucleus into the anterior chamber.

Hydrodelineation was performed to decrease the size of the nucleus. When the nucleus failed to dislocate into the anterior chamber it was tumbled out of the bag using an irrigating vectis.

Once the nucleus was free of the bag a $4 \mathrm{~mm}$ tapered irrigating vectis was positioned behind it and, with the ACM running, was drawn into the tunnel. The fragments of the nucleus were broken off within the tunnel by a tilting action of the vectis. The removal of the nucleus portions through the tunnel was facilitated by the positive intraocular pressure generated by the ACM and also by the push of the vectis 
against the posterior wall of the tunnel. The remaining cortex was aspirated using a cortex extractor mounted on a $5 \mathrm{ml}$ syringe introduced through the side port. The capsule was kept taut by the positive intraocular pressure generated by the ACM, thereby reducing the chances of a posterior capsule rupture. A $5.5 \mathrm{~mm}$ optic PMMA lens (Pharmacia 812C) was implanted into the bag in all cases. The external incision was closed with a box suture of 10/0 vicryl. All ports were closed with stromal hydration. The conjunctiva was secured in place with cautery.

When the operation was combined with a trabeculectomy, the scleral tunnel was converted into a flap, a small portion of deep sclera $(2 \times 3 \mathrm{~mm})$ was excised from the posterior lip and a basal broad iridectomy was performed through the opening. The scleral flap and the conjunctiva were secured with interrupted $10 / 0$ vicryl sutures.

\section{Results \\ Demography}

Of the 300 patients entered into this study 192 were female and 108 were male ( $64 \%$ vs $36 \%$ ). The mean age at the time of surgery was 74 years (range 24-94 years). The best visual acuity in the better eye gives an indication of visual impairment in daily life at the time of admission. Sixty-four per cent of patients below the age of 75 years had good vision in the better eye and were still within the legal requirement for driving (6/6-6/12), in comparison with $55 \%$ who were above the age of 75 years. Twentyone per cent of patients had age-related macular degeneration, $7 \%$ had diabetic retinopathy (21 patients), $8 \%$ had glaucoma (24 patients), 5 patients had amblyopia and $9 \%$ had other pathologies (27 patients), as shown in Table 1.
One hundred and seventeen operations were done as day cases, and 183 as inpatient procedures. All patients who had either combined operations (cataract extraction with IOL implantation and trabeculectomy; 15 patients) or bilateral simultaneous cataract surgery (14 patients) were kept in hospital overnight.

Two hundred and forty-nine eyes were treated under local anaesthesia (peribulbar/retrobulbar/sub-Tenon's injection) and 51 eyes were treated under general anaesthesia. All simultaneous bilateral cataract extractions were performed under general anaesthesia. Two hundred and eighty-seven cases were performed by one of us (T.W.); the remainder were done by training grades.

Ninety-nine patients had a pseudophakos in the other eye. No pre-operative antibiotics were given to any of the patients. Patients operated on prior to January 1998 received topical chloramphenicol drops only (134 patients) at the end of surgery, while those who were operated on after January 1998 had a subconjunctival injection (cefuroxime $125 \mathrm{mg}$ or gentamicin $20 \mathrm{mg}$ ). All 14 patients who underwent bilateral simultaneous cataract surgery had a subconjunctival injection of one of the aforementioned antibiotics.

\section{Complications (Table 2)}

Twenty-two patients (7\%) had some intraoperative complication in the form of Descemet's tear $(0.6 \%)$, iris prolapse $(2.6 \%)$, or posterior capsule rupture alone $(2.2 \%)$ or with vitreous loss $(1 \%)$ and requiring an anterior chamber IOL in $0.6 \%$ of eyes. In no case were fragments of the nucleus lost in the vitreous.

Sixty-one patients $(20.3 \%)$ had some type of immediate post-operative complication (within $24 \mathrm{~h}$ ). Recordable corneal oedema was seen in 56 (18.6\%) eyes; it resolved in a majority of the cases with the exception of

Table 1. Ocular pathology on admission

\begin{tabular}{lcc}
\hline Type of pathology & No. of patients & \% known pathology \\
\hline None present & 160 & 53.3 \\
Age-related maculopathy & 3 & 21.0 \\
Drusen/RPE changes & $(55)$ & $(18.3)$ \\
Disciform & $(8)$ & $(2.7)$ \\
Diabetic retinopathy & 21 & 7.0 \\
Background & $(14)$ & $(4.7)$ \\
Proliferative & $(7)$ & $(2.3)$ \\
Maculopathy & $(0)$ & $(0.0)$ \\
Glaucoma & 24 & 8.0 \\
Amblyopia & 5 & 1.7 \\
Other & 27 & 9.0 \\
Myopia/myopic retinal degeneration & $(12)$ & $(4.0)$ \\
Corneal opacities & $(4)$ & $(1.4)$ \\
Lids & $(3)$ & $(1.0)$ \\
Retinal detachment repair & $(3)$ & $(1.0)$ \\
Retinitis pigmentosa & $(2)$ & $(0.7)$ \\
Asteroid hyalosis & $(1)$ & $(0.3)$ \\
Vascular occlusion & $(1)$ & $(0.3)$ \\
Malignant melanoma & $(1)$ & $(0.3)$ \\
Total & 300 & 100 \\
\hline
\end{tabular}

RPE, retinal pigment epithelium. 
Table 2. Incidence of complications

\begin{tabular}{lcc}
\hline Type of complication & No. with complications & $\%$ total \\
\hline Intra-operative period & 22 & 7 \\
Capsular complications & 7 & 2.2 \\
Capsular complications with vitreous loss & 3 & 1.0 \\
Capsular loss with vitreous loss - anterior chamber IOL & 2 & 0.6 \\
Iris prolapse & 8 & 2.6 \\
Tear in Descemet's membrane & 2 & 0.6 \\
Immediately post-operatively (within 24 h) & 61 & 20.3 \\
Corneal oedema & 56 & 18.6 \\
Raised IOP & 5 & 1.7 \\
At first outpatient follow-up visit (within 14 days) & 8 & 2.6 \\
Endophthalmitis & 3 & 1.0 \\
Raised IOP & 2 & 0.6 \\
Corneal oedema & 3 & 1.0 \\
Complications at 3 months follow-up & 46 & 15 \\
Corneal oedema & 3 & 1.0 \\
Optic atrophy & 1 & 0.2 \\
Posterior capsular thickening (requiring capsulotomy) & 40 & 13.3 \\
Raised IOP & 2 & 0.5 \\
\hline
\end{tabular}

3 eyes. One of these patients died within 8 months of operation, one had a scarred cornea pre-operatively and the other had a penetrating keratoplasty done for the same reason. High intraocular pressure (IOP) was seen in $5(1.7 \%)$ eyes; it responded well to topical and oral antiglaucoma medication.

Eight patients $(2.6 \%)$ had a persistent or a new complication when seen at the first follow-up, which was at 14 days post-operatively. Raised IOP and corneal oedema persisted in $2(0.6 \%)$ and $3(1.0 \%)$ eyes respectively. Endophthalmitis was seen in $3(1.0 \%)$ eyes at this follow-up. Treatment with sensitive antibiotics helped in achieving good vision in 2 patients. One patient developed optic atrophy and had a visual acuity of $6 / 36$ in the affected eye at the last follow-up.
Forty patients $(13.3 \%)$ had a significant posterior capsular opacification warranting a YAG capsulotomy. All the patients who underwent a combined operation had some degree of hyphaema, which ranged from microscopic to one-third of the anterior chamber. The hyphaema resolved in all these patients in 1-14 days.

\section{Post-operative acuity}

Two hundred and seventy-one patients (90\%) showed a gain of 1, 2, 3 or more lines of Snellen acuity at the 3 month follow-up (Fig. 1). In 23 patients (7.3\%) the visual acuity remain unchanged and in 6 patients $(2 \%)$ it was worse compared with their pre-operative visual status.

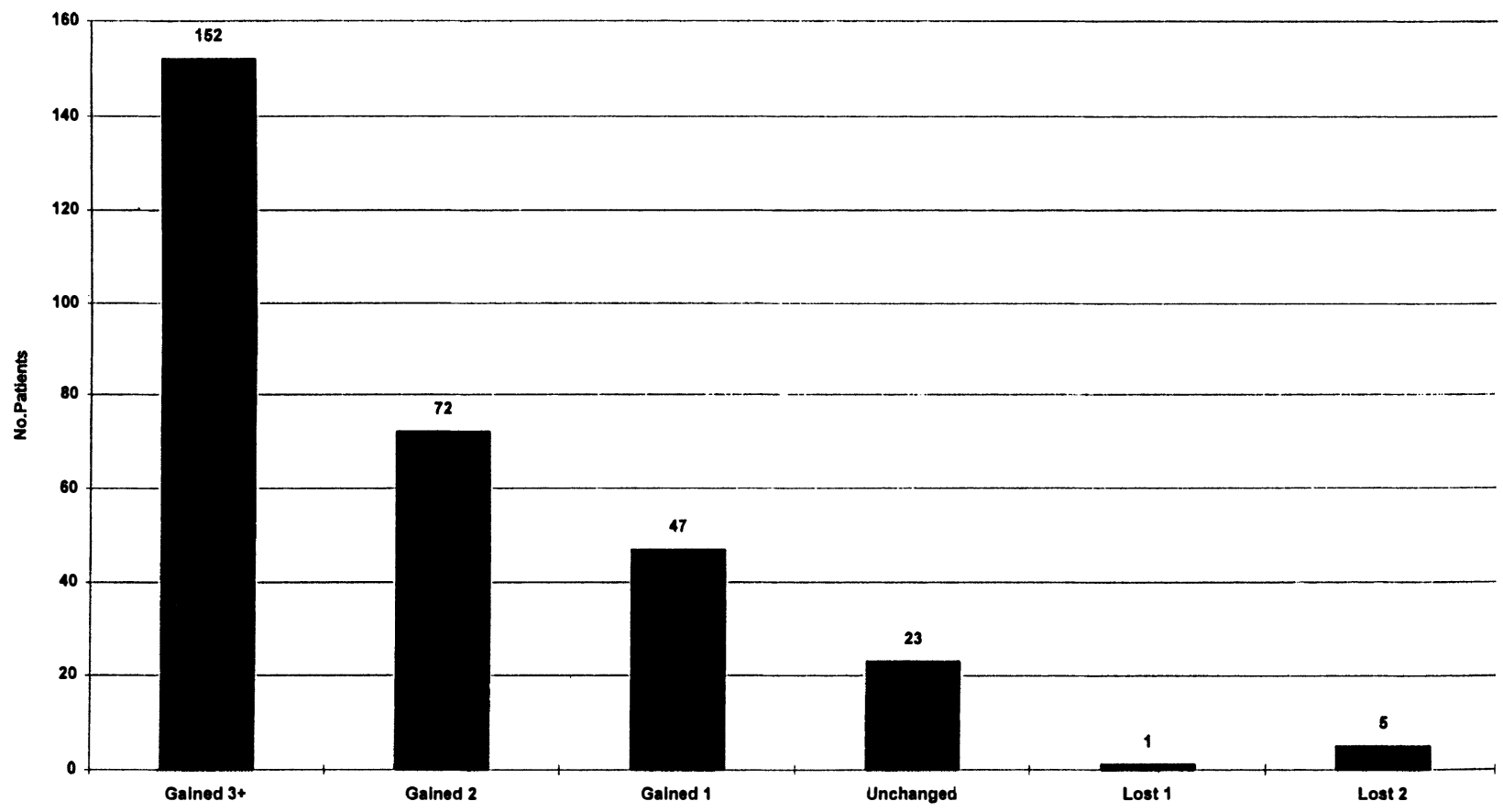

Fig. 1. Visual acuity gain/loss (Snellen lines) at 3 months post-operatively. 


\section{Discussion}

Cataract extraction with implantation of an IOL using an anterior chamber maintainer was introduced by Blumenthal. Though phacoemulsification has overtaken it as a safe option for a small-incision technique, the ACM holds its place as it is technology-independent and does not require special instrumentation.

We have used a $6 \mathrm{~mm}$ scleral incision as it has not been found to be different from a $3 \mathrm{~mm}$ incision in terms of induced astigmatism at 3 months post-operatively, as reported by Gills et al., ${ }^{3}$ Martin et al. ${ }^{4}$ found no significant difference in the post-operative inflammation when comparing a $3 \mathrm{~mm}$ with a $6 \mathrm{~mm}$ incision. A $6 \mathrm{~mm}$ incision allows easy introduction of the vectis and prolapsing the nucleus out of the bag.

A tear in Descemet's membrane was noted in 2 patients at the end of surgery. These most probably occurred during the capsulorrhexis while trying to get hold of the loose anterior capsular flap in the currents of the ACM, and during placement of the receding haptic when inserting the lens into the bag.

At least $50 \%$ of patients showed superior corneal oedema on first dressing, which was severe enough to be recorded in the patient's notes in just under $20 \%$.

Endothelial loss occurs in a number of ways, of which the following are most significant: (1) The three-step tunnel incision used for this procedure has been shown by Lavery et al. ${ }^{5}$ to have an increased endothelial cell loss compared with a two-step procedure. The internal opening of the tunnel is through corneal stroma and causes direct damage to the endothelium. (2) As the nucleus is brought towards the tunnel it frequently brushes against the superior endothelium. The pressure exerted by the vectis during fragmentation leads to further endothelial loss.

Though the corneal oedema resolved in the majority of cases, it persisted in 3 patients one of whom required a penetrating keratoplasty. Meticulous clinical assessment and documentation of the corneal endothelium prior to surgery is likely to reduce this complication further. An endothelial specular cell counter is not available in our department.

Iris prolapse was seen in 8 eyes and occurred mostly during the learning curve of this surgery. It occurs if the anterior chamber is entered in the limbal area rather than through the corneal stroma so that the iris root is in the same plane as the tunnel and is easily pushed out by the positive IOP.

Endophthalmitis was seen in 3 patients (2.2\%), all within the first 9 months of the surgery, in comparison with $0.3 \%$ as reported in the NCSS. As the ACM is placed into an opening in the inferotemporal segment of the limbus and remains there for about 15-20 min, the opening is in close proximity to the lower eyelid. On removing the lid speculum, fluid contaminated by lid organisms is drawn into the anterior chamber leading to bacterial overload, ${ }^{6}$ and this we presume is the cause of infection. Two of the 3 patients affected achieved their visual potential and the third developed optic atrophy.

Posterior capsule rupture with or without vitreous loss was seen in 12 cases. Two of these patients required an anterior chamber IOL. This complication occurred during mobilisation of the nucleus into the anterior chamber and was related to zonular dehiscence.

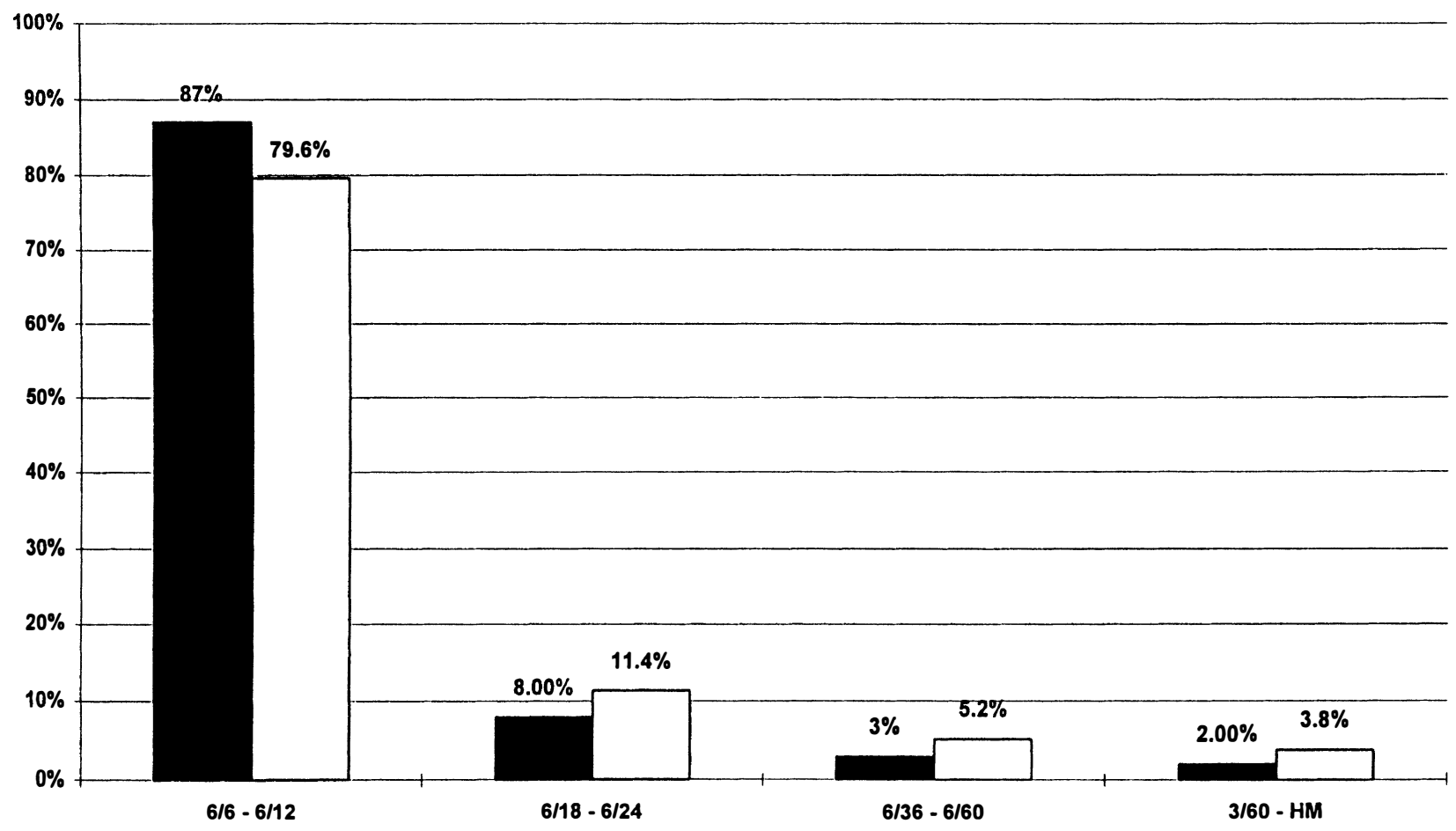

Fig. 2. Visual acuity at 3 months post-operatively. Black columns, present study; open columns, National Cataract Surgery Survey. 
Dislocation of the nucleus into a small capsulorrhexis can also put stress on zonules and may have been an additional cause.

Capsular opacification occurred in a significant proportion of cases and at the time of this review (maximum 20 month follow-up) 13.3\% required a YAG laser capsulotomy. In none has this led to a secondary complication. A similar percentage (13\%) was reported by the NCSS. ${ }^{7}$

Eighty-seven per cent of our patients gained a visual acuity of $6 / 6-6 / 12$, in comparison with $79.6 \%$ as reported by the NCSS. A comparison of post-operative visual acuity in our series and the NCSS is shown in Fig. 2.

Small-incision cataract surgery using an ACM has been shown to be an acceptable surgical procedure and all complications found in our assessment of 300 consecutive cases are within the range of complications found by the NCSS, with the exception of corneal endothelial decompensation and endophthalmitis. We do not think the technique is suitable for patients with clinical evidence of corneal endothelial cell reduction.
Good wound closure and subconjunctival antibiotics have been shown to deal with the increased risk of infection.

\section{References}

1. Blumenthal M, Moisseiev J. Anterior chamber maintainer for extracapsular cataract extraction and intraocular lens implantation. J Cataract Refract Surg 1997;13:204-6.

2. Koch PS. Mastering phacoemulsification. Thorofare, NJ: Slack, 1994.

3. Gills J, et al. Use of small incisions to control astigmatism and inflammation following cataract surgery. J Cataract Refract Surg 1991;17:740-4.

4. Martin RG, et al. Effect of small incision intraocular lens surgery on postoperative inflammation and astigmatism. J Cataract Refract Surg 1992;18:51-7.

5. Lavery KT, et al. Endothelial cell loss after $4 \mathrm{~mm}$ cataract surgery. J Cataract Refract Surg 1995;21:305-8.

6. Montan Per G, et al. Endophthalmitis after cataract surgery: risk factors relating to technique and events of the operation and patient history. Ophthalmology 1998;105:2171-7.

7. Desai P. The National Cataract Surgery Survey. II. Clinical outcomes. Eye 1993;7:489-94. 